\title{
Patient Perception following Alloplastic Total Temporomandibular Joint Replacement
}

\author{
Jameel Desai \\ Department of Maxillofacial and Oral Surgery, Oral and Dental Hospital, University of Pretoria, Pretoria, South Africa
}

\section{Abstract}

Introduction: A retrospective analysis was done on patients who had undergone unilateral temporomandibular joint reconstruction using alloplastic temporomandibular prostheses. Aim: The study wished to highlight patient perceptions after surgery; specifically relating to pain, function, and aesthetics. Material and Method: Twenty three patients were assessed using a visual analogue scale (VAS) for pain, ability to eat, quality of life and scar appraisal. Results: Patients Vas scores were favourable for all parameters save for scar appreciation. Conclusion: Total temporomandibular alloplastic joint replacement is a viable treatment option, with generally positive surgical outcomes for patients.

Keywords: Alloplastic prosthesis, patient perception, temporomandibular joint replacement

\section{INTRODUCTION}

The use of alloplastic prostheses for the reconstruction of the temporomandibular joint (TMJ) is well documented. ${ }^{[1-3]}$ Orthopedic surgeons have been replacing knee and hip joints with a host of alloplastic materials for many decades, and maxillofacial surgeons have also been doing total TMJ replacements for well over 50 years; however, these TMJ replacements have only been done in South Africa for about 15 years now. There has been very little feedback in the South African setting, regarding the perception that patients have following such replacement surgeries. A series of 23 patients who received unilateral total alloplastic TMJ replacements from 2008 to 2012 are presented.

\section{Methods}

Patients were consulted and agreed to participate in this study.

The patients selected for inclusion had to meet the following criteria:

The patients were selected on the basis of having internal joint derangement as per Wilkes classification (Stages IV and V).

They were all consulted, operated on, and followed up by the author while in independent private practice at Mediclinic Hospital, Newcastle, South Africa. All relevant findings were documented by the author.

\begin{tabular}{|l|l|}
\hline \multicolumn{2}{|c|}{ Access this article online } \\
\hline Quick Response Code: & Website: \\
\hline & www.amsjournal.com \\
\cline { 2 - 3 } & \\
\hline
\end{tabular}

Patients who received joint replacements for any other cause (trauma, pathology, etc.) were not included in this study. Every patient was followed up at 2, 6, and 12 weeks postoperatively, and the final interview being conducted at 24 -week postoperatively. Patients were questioned using a $10 \mathrm{~cm}$ visual analog scale (VAS).

Patients were asked to rate their ability to chew; pain levels; scar appreciation; and overall quality of life (QOL), after the surgery. The VAS grade was as follows:

0 - Worst possible perception, 3 - below average perception, 5 -average perception, 8 - above average perception, 10 - best possible perception.

This study also included parameters such as: gender, side bias, mean age and preand post-operative mouth opening. All patients received a standardized surgical protocol involving general anesthesia and local anesthetic infiltration. The surgery [Figures 1-5] always involved 2 surgical approaches, that is, a submandibular and modified preauricular access. Condylectomy without coronoidectomy was performed, and the prosthesis inserted (TMJ stock prosthesis from Zimmer Biomet CMF, Jacksonville, FL, USA). All wounds were closed and dressed in the same way, and postoperative medication standardized, but only altered in the case of drug allergies.

Address for correspondence: Dr. Jameel Desai Postnet Suite 116, P/Bag X6603, Newcastle 2940, South Africa. E-mail: drdesai@maxfac.co.za

This is an open access journal, and articles are distributed under the terms of the Creative Commons Attribution-NonCommercial-ShareAlike 4.0 License, which allows others to remix, tweak, and build upon the work non-commercially, as long as appropriate credit is given and the new creations are licensed under the identical terms.

For reprints contact: reprints@medknow.com

How to cite this article: Desai J. Patient perception following alloplastic total temporomandibular joint replacement. Ann Maxillofac Surg 2018;8:83-5. 


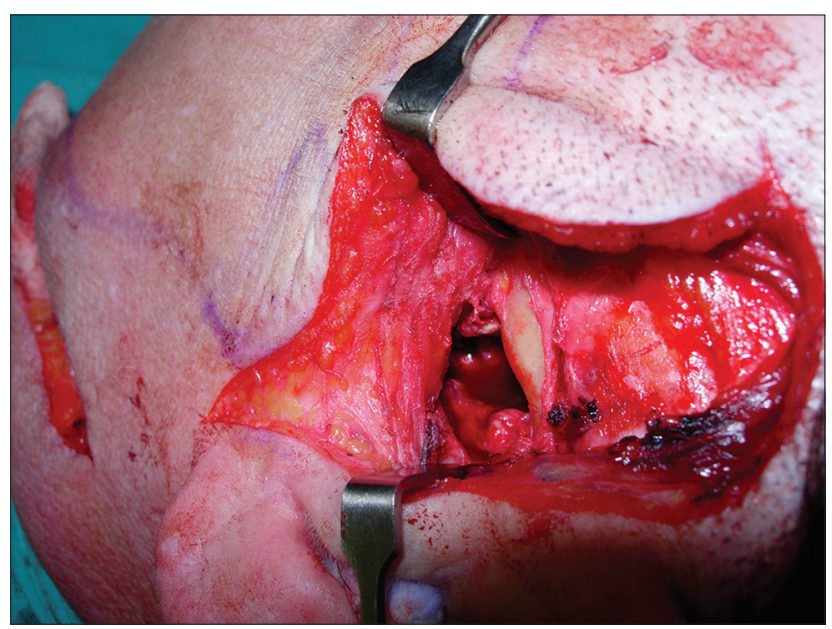

Figure 1: Preauricular approach with gap arthroplasty. The condylectomy has been performed. The zygomatic arch is seen, as are the fibers of the temporalis muscle

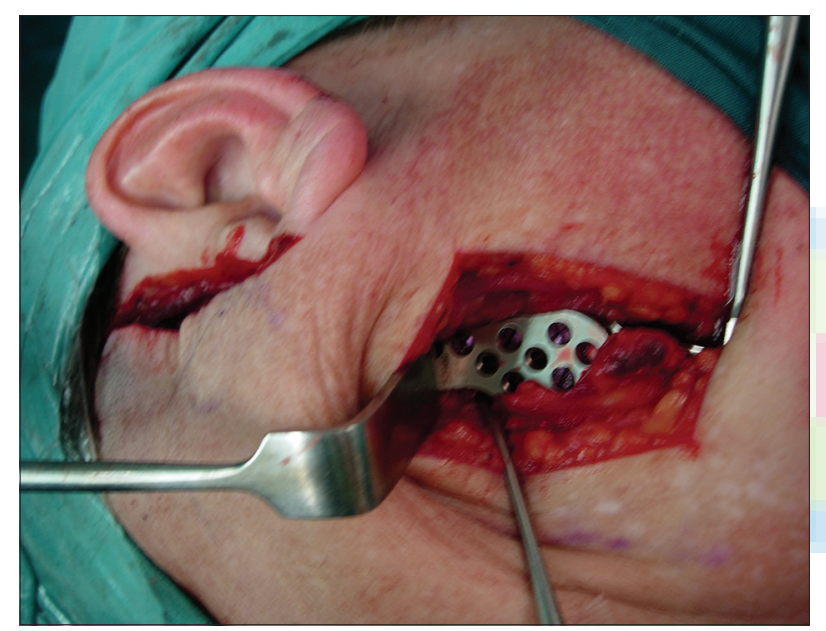

Figure 3: The mandibular (condyle) component can be seen screwed to the ramus through the submandibular incision

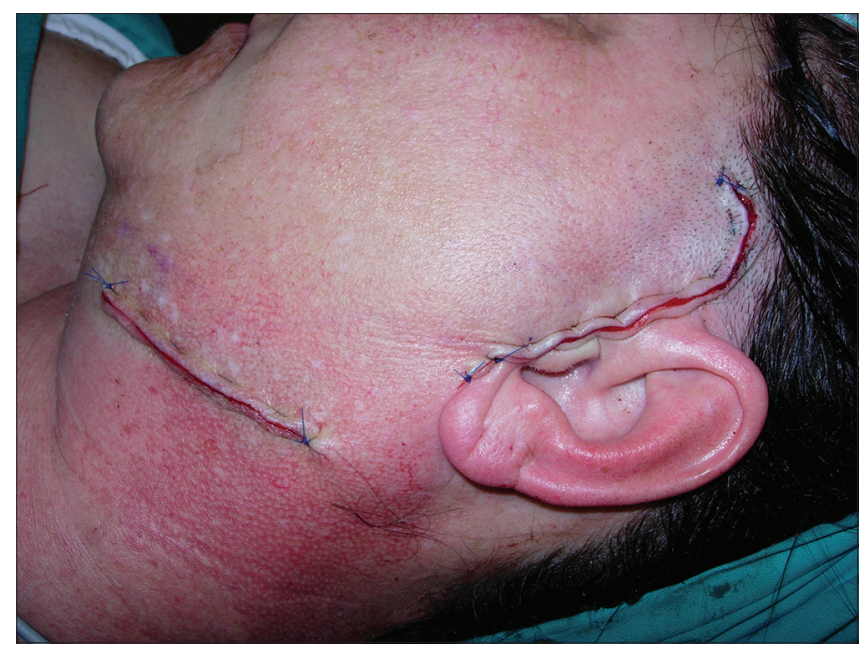

Figure 5: Wound closures standardized in all cases to 4.0 Prolene skin sutures placed using a vertical mattress orientation

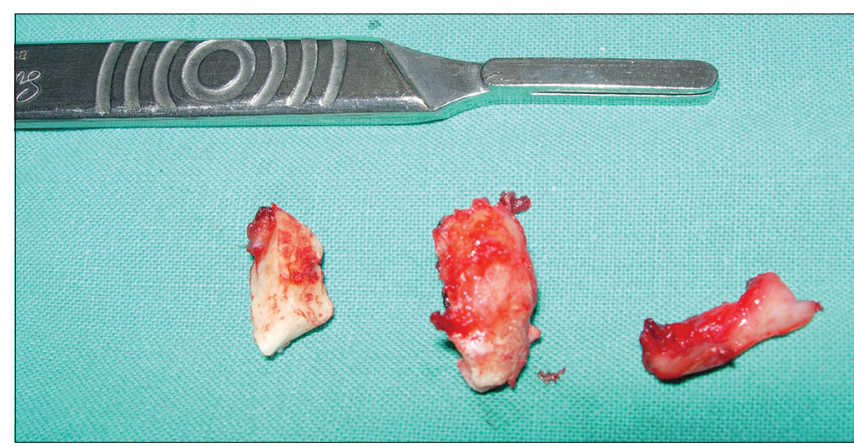

Figure 2: The condylar head and articular disc (removed in 2 pieces) are seen. Note the eroded superior articulating surface of the condylar head (center)

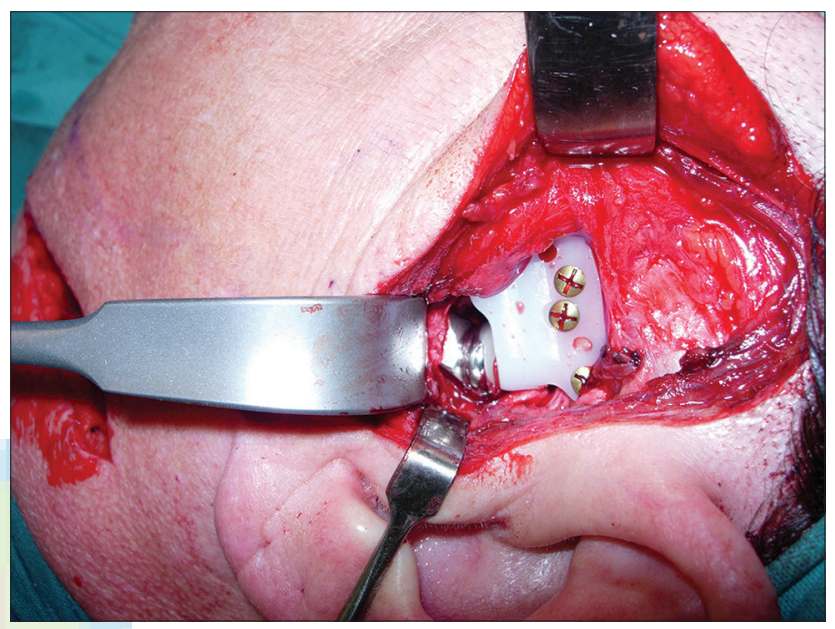

Figure 4: Fossa and condyle components can be seen articulating in situ

\section{RESULTS}

A total of 23 patients were included in this audit, of which 21 were female and 2 were male. The patient ages ranged from 19 years to 67 years, with the mean age being 54 years. Of the total 23 joint replacements done, 17 of those were on the patients' right side; and only 6 were done on the left. The preoperative and postoperative mouth opening was also recorded in millimeters, as the maximal incisal opening (MIO). The mean preoperative (MIO) was $21 \mathrm{~mm}$, while the mean postoperative distance increased to $33 \mathrm{~mm}$. Patients were questioned about their perceptions regarding the outcome of the surgery, in respect of their ability to eat, their levels of joint pain, the appearances of the surgical scars, and their overall QOL since the surgery. The following graph depicts the VAS scores pertaining to those questions [Figure 6].

A record of surgical complications was also kept and is documented below.

Of the total number of patients, 12 patients had transient palsies of CNVII (marginal mandibular and/or frontal branches). One patient presented with a CNV (inferior alveolar nerve [IAN]) paresthesia at 24 weeks, and there was 1 case of localized wound sepsis. One case of chronic pain was reported at 24 weeks, and 2 cases were documented to have intraoperative hemorrhage, that was controlled at the time of surgery. 


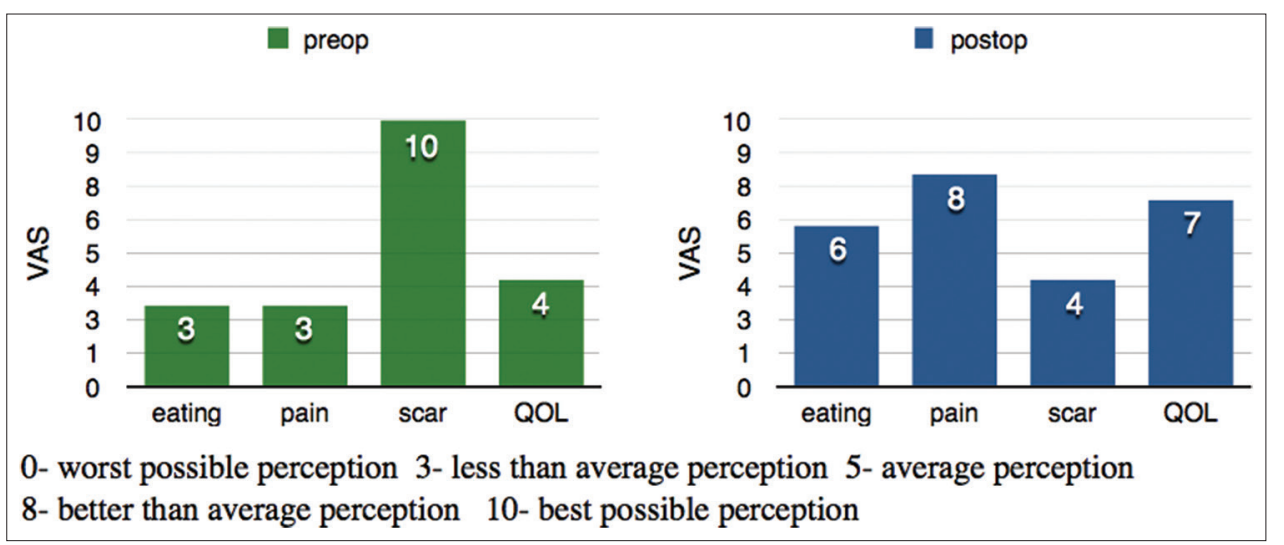

Figure 6: Preoperative and postoperative graphs depicting the visual analog scale scores

\section{DISCUSSION}

The use of alloplastic TMJ prostheses has steadily been gaining momentum as a viable and predictable solution for the replacement of degenerated joints that are refractory to conservative or less invasive interventions. South African maxillofacial surgeons seem to be more resistant, albeit circumspect in utilizing these prostheses in their patients. It is speculated that this is due to the lack of exposure to such procedures, as it has only recently started gaining momentum. Perhaps, the surgeons are unsure as to what patient expectations might be following such surgeries? It is hoped that this audit of patients will hopefully encourage surgeons to utilize alloplastic TMJ replacement surgery as a legitimate and effective means of treatment for advanced degenerative damage to the joint. ${ }^{[4]}$ Further, it is hoped that general dental practitioners would also be encouraged to advise and refer their patients without fear of negative outcomes regarding alloplastic total joint replacement procedures. What is evident from this audit, is that patient perceptions with respect to pain, mastication, and overall QOL seems to be improved following such surgery; a trend reported by Leandro et al. after their analysis of a large sample size over a 10 -year period of follow-up. ${ }^{[5]}$ What is particularly encouraging is the improvement in mouth opening; also seen by other surgeons. ${ }^{[6]}$ It is the improvement of mouth opening and decrease in pain intensity that gives patients a better overall masticatory ability, and presumably a better QOL. Almost every patient reported no regrets to have had the surgery performed, but most indicated that the first 6 weeks after surgery were extremely challenging. The surgical scars were also not of major concern for patients, as these may be very strategically placed within skin resting lines. The intra- and postoperative complications were transient and resolved over time, save for the CNV (IAN) paresthesia which had not. If a surgical protocol and postoperative physiotherapy are standardized and adhered to, then results appear to be more predictable. ${ }^{[7,8]}$ Obviously, due to the sample size, no meaningful statistical analysis could be conducted. Nonetheless, this qualitative evaluation of outcomes addressed most patients' concern following surgery and could be considered as relevant.

With a growing number in our series of cases, it is hoped that more analytical data should emerge from further papers on the subject.

\section{ConcLusion}

The use of alloplastic total joint replacement prostheses are viable, and present favourable outcomes for both patients and clinicians alike. From this study, it should become apparent that the phobia surrounding their use is not warranted.

\section{Declaration of patient consent}

The authors certify that they have obtained all appropriate patient consent forms. In the form the patient(s) has/have given his/her/their consent for his/her/their images and other clinical information to be reported in the journal. The patients understand that their names and initials will not be published and due efforts will be made to conceal their identity, but anonymity cannot be guaranteed.

\section{Financial support and sponsorship}

Nil.

\section{Conflicts of interest}

There are no conflicts of interest.

\section{References}

1. Burel JP, Van Reck J. Temporomandibular joint prosthesis. A retrospective study from 1947. Acta Stomatol Belg 1997;94:9-21.

2. DriemelO, Braun S, Müller-Richter UD, Behr M, Reichert TE, Kunkel M, et al. Historical development of alloplastic temporomandibular joint replacement after 1945 and state of the art. Int J Oral Maxillofac Surg 2009;38:909-20.

3. Jones RH. Temporomandibular joint reconstruction with total alloplastic joint replacement. Aust Dent J 2011;56:85-91.

4. Sidebottom AJ. Alloplastic or autogenous reconstruction of the TMJ. J Oral Biol Craniofac Res 2013;3:135-9.

5. Leandro LF, Ono HY, Loureiro CC, Marinho K, Guevara HA. A ten-year experience and follow-up of three hundred patients fitted with the Biomet/Lorenz Microfixation TMJ replacement system. Int J Oral Maxillofac Surg 2013;42:1007-13.

6. Westermark A. Total reconstruction of the temporomandibular joint. Up to 8 years of follow up of patients treated with Biomet ${ }^{\mathrm{TM}}$ total joint prostheses. Int J Oral Maxillofac Surg 2011;40:366-71.

7. Sidebottom AJ, Gruber E. One-year prospective outcome analysis and complications following total replacement of the temporomandibular joint with the TMJ concepts system. Br J Oral Maxillofac Surg 2013;51:620-4.

8. Mercuri LG, Edibam NR, Giobbie-Hurder A. Fourteen-year follow-up of a patient-fitted total temporomandibular joint reconstruction system. J Oral Maxillofac Surg 2007;65:1140-8. 